\title{
Measurement of azimuthal asymmetries of the unpolarized cross section at HERMES
}

Francesca Giordano, Rebecca Lamb, and HERMES Collaboration

Citation: AIP Conference Proceedings 1149, 423 (2009); doi: 10.1063/1.3215681

View online: https://doi.org/10.1063/1.3215681

View Table of Contents: http://aip.scitation.org/toc/apc/1149/1

Published by the American Institute of Physics

Articles you may be interested in

Azimuthal asymmetries in DVCS on unpolarized hydrogen and deuterium targets

AIP Conference Proceedings 1149, 619 (2009); 10.1063/1.3215724

\section{Conference Proceedings}

\section{Get $30 \%$ off all print proceedings!}

Enter Promotion Code PDF-30 at check 


\title{
Measurement of azimuthal asymmetries of the unpolarized cross section at HERMES
}

\author{
Francesca Giordano* and Rebecca Lamb (on behalf of the HERMES \\ Collaboration $)^{\dagger}$
}

${ }^{*} I N F N \&$ Università degli studi di Ferrara, giordano@fe.infn.it

$\dagger$ University of Illinois, rlamb2@illinois.edu

\begin{abstract}
A multi-dimensional $\left(x, y, z, P_{h}\right)$ extraction of $\cos \phi_{h}$ and $\cos 2 \phi_{h}$ azimuthal asymmetries of unpolarized Semi-Inclusive Deep Inelastic Scattering at HERMES is discussed. The use of data taken with hydrogen and deuterium targets and the separation of positive and negative hadrons allow to access flavor-dependent information about quark intrinsic transverse momenta and spinorbit correlations. This flavor sensitivity allows for a discrimination between theoretical models in the HERMES kinematic regime.
\end{abstract}

Keywords: Semi-Inclusive DIS; Azimuthal asymmetries; Intrinsic transverse momentum and spin. PACS: $13.88 .+\mathrm{e}, 13.60 .-\mathrm{r}$

\section{INTRODUCTION}

In Deep Inelastic Scattering (DIS), the structure of the nucleon is probed by the interaction of a high energy lepton and a target nucleon, via the exchange of a virtual boson. If at least one of the produced hadrons is detected in coincidence with the scattered lepton, the reaction is called Semi-Inclusive Deep Inelastic Scattering (SIDIS):

$$
l(\mathbf{k})+N(\mathbf{P}) \rightarrow l^{\prime}\left(\mathbf{k}^{\prime}\right)+h\left(\mathbf{P}_{h}\right)+X\left(\mathbf{P}_{X}\right),
$$

where $l\left(l^{\prime}\right)$ is the incident (scattered) beam lepton, $N$ is the target nucleon, $h$ the detected hadron and $X$ the target remnant. The quantities in parentheses are the corresponding four-momenta.

If the cross section is unintegrated over the hadron momentum component transverse to the virtual photon direction $P_{h \perp}$ (Fig. 1), an azimuthal dependence around the outgoing hadron direction exists [1]:

$$
\begin{aligned}
\frac{d \sigma}{d x d y d z d P_{h \perp}^{2} d \phi_{h}}= & \frac{\alpha^{2}}{x y Q^{2}}\left(1+\frac{\gamma^{2}}{2 x}\right)\left\{A(y) F_{U U, T}+B(y) F_{U U, L}+\right. \\
& \left.C(y) \cos \phi_{h} F_{U U}^{\cos \phi_{h}}+B(y) \cos 2 \phi_{h} F_{U U}^{\cos 2 \phi_{h}}\right\},
\end{aligned}
$$

where $\phi_{h}$ is the azimuthal angle of the hadron plane around the virtual-photon direction (Fig. 1). Here $Q^{2}$ and $y$ are respectively the negative squared four-momentum and the fraction of the lepton's energy transferred to the virtual photon, $x$ is the Bjorken scaling variable and $z$ is the virtual photon's fractional energy transferred to the produced hadron. For the structure functions $F$, the subscript $U U$ denotes Unpolarized beam and

CP1149, Spin Physics, $18^{\text {th }}$ International Spin Physics Symposium

edited by D. G. Crabb, D. B. Day, S. Liuti, X. Zheng, M. Poelker, and Y. Prok

(C) 2009 American Institute of Physics 978-0-7354-0686-5/09/\$25.00 


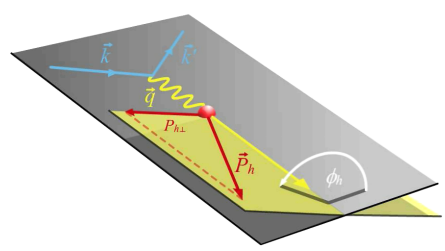

FIGURE 1. Definition of the azimuthal angle $\phi_{h}$ between the scattering plane (grey) and the hadron production plane (yellow).

Unpolarized target, $T(L)$ indicates the Transverse (Longitudinal) polarization of the virtual photon, $\alpha$ is the electromagnetic coupling constant, $\gamma=2 M x / Q$ with $M$ the target mass, $A(y) \approx\left(1-y+1 / 2 y^{2}\right), B(y) \approx(1-y)$ and $C(y) \approx(2-y) \sqrt{1-y}$.

Two mechanisms are expected to give important contributions to the azimuthal dependence of the unpolarized cross section in the hadron transverse momentum range accessed at HERMES:

- the Cahn effect, a pure kinematic effect, generated by the non-zero intrinsic transverse motion of quarks, already pointed out by R. Cahn in 1978 [2];

- the Boer-Mulders effect, introduced more recently (1997) by D. Boer and P. J. Mulders [3]; this mechanism originates from a coupling between quark transverse momentum and quark transverse spin.

\section{The HERMES experiment}

The fixed target HERMES experiment was operated for more than 10 years until 2007 at the electron-positron storage ring of HERA at DESY. The HERMES spectrometer [4] was a forward angle instrument consisting of two symmetric halves (top, bottom) above and below the horizontal plane. It was characterized by very high efficiency $(98 \div 99 \%)$ in electron-hadron separation, provided by a transition radiation detector, a preshower scintillation counter and an electromagnetic calorimeter. In addition, a dual-radiator Ring-Imaging CHerenkov detector (RICH) provided good hadron-type identification for momenta above $2 \mathrm{GeV} / \mathrm{c}$.

\section{MULTI-DIMENSIONAL UNFOLDING AND RESULTS}

The cross section azimuthal modulations can be measured via the $\left\langle\cos n \phi_{h}\right\rangle$-moments:

$$
\left\langle\cos n \phi_{h}\right\rangle=\frac{\int \cos n \phi_{h} d^{5} \sigma}{\int d^{5} \sigma},
$$

where $n=1,2$ and $\int d^{5} \sigma$ stands for $\int d x d y d z d P_{h \perp}^{2} d \phi_{h} \frac{d^{5} \sigma}{d x d y d z d P_{h \perp}^{2} d \phi_{h}}$.

The extraction of these cosine moments from data is challenging because they couple to a number of azimuthal modulations that are due to experimental sources, e.g. detector 

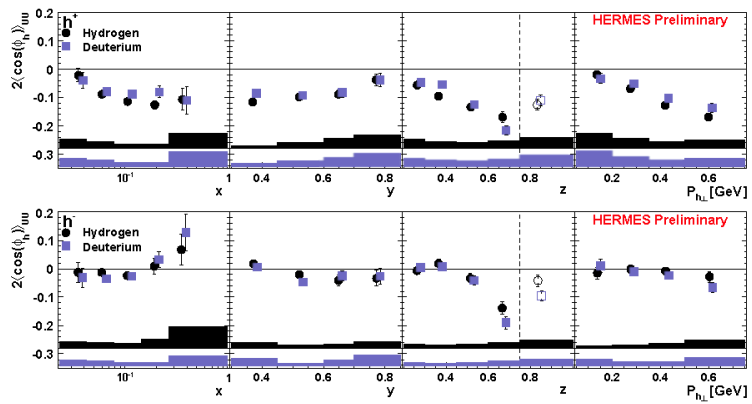

FIGURE 2. The $\cos \phi_{h}$ moments for positive (upper panel) and negative (lower panel) hadrons, extracted from hydrogen (circles) and deuterium (squares) data, shown as projection versus the kinematic variables $x, y, z$ and $P_{h \perp}$.

geometrical acceptance and higher-order QED effects (radiative effects). Moreover, typically the event sample is binned only in one variable (1-dimensional analysis), and integrated over the full range of all the other ones, while the structure functions $F$ used in equation 2 and the instrumental contributions depend on all the kinematic variables $x$, $y, z$ and $P_{h \perp}$ simultaneously.

Therefore, in order to determine the cosine moments corrected for radiative and detector smearing, an unfolding procedure [6] was used, in which the event sample is binned simultaneously in a1l the relevant variables (multi-dimensional analysis ${ }^{1}$ ).

The unfolding algorithm is based on the relation between the unknown distribution of Born yields $B(j)$ and the distribution of measured yields $X(i)$ :

$$
X(i)=\sum_{j=1}^{n_{b}} S(i, j) B(j)+\beta(i) .
$$

where $n_{b}$ is the total number of bins and $\beta(i)$ is a vector that contains the events smeared into the measured sample from outside the acceptance. The Smearing matrix $S(i, j)$ describes the probability that an event originating from the Born bin $j$, corresponding to the original kinematics (free from experimental distortions), is actually observed in the measured bin $i$. Both the background $\beta(i)$ and the smearing matrix $S(i, j)$ are determined by a detailed Monte Carlo simulation of the experimental apparatus.

Assuming a non-singular $S(i, j)$ matrix one obtains:

$$
B(j)=\sum_{i=1}^{n_{b}} S^{-1}(j, i)[X(i)-\beta(i)] .
$$

The extraction of cosine moments from the Born yields $B(j)$ can be performed by linear regression that takes into account the correlations between bins introduced by the smearing. In this way one pair of moments can be obtained in each kinematic bin $\left(\left\langle\cos \phi_{h}\right\rangle,\left\langle\cos 2 \phi_{h}\right\rangle\right)$, which represents results that are fully differential in all variables.

\footnotetext{
${ }^{1}$ For a more detailed discussion about 1 - and multi-dimensional analysis see [5].
} 

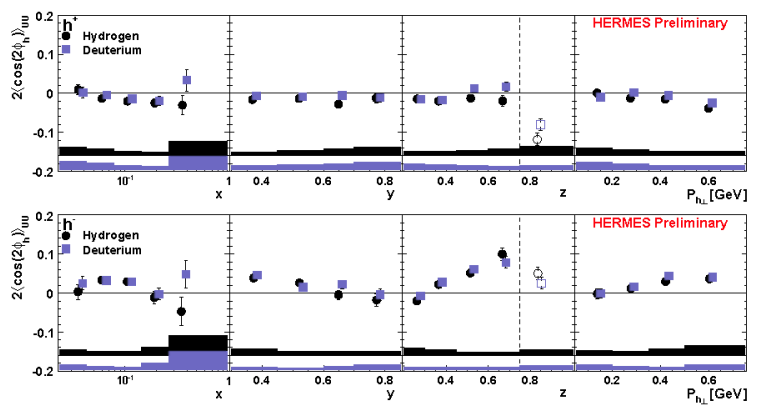

FIGURE 3. The $\cos 2 \phi_{h}$ moments for positive (upper panel) and negative (lower panel) hadrons, extracted from hydrogen (circles) and deuterium (squares) data, shown as projection versus the kinematic variables $x, y, z$ and $P_{h \perp}$.

The dependence of a moment on a single variable can be obtained by projecting the fully differential result onto the variable under study by weighting the moment in each bin $k$ with the corresponding unpolarized $4 \pi$ cross section $\sigma_{k}^{4 \pi}$ (defined by a Monte Carlo), for instance in case of the $\ell$-th $x$-bin: $\left\langle\cos n \phi_{h}\right\rangle\left(x_{\ell}\right)=\sum_{k} \sigma_{k}^{4 \pi}\left\langle\cos n \phi_{h}\right\rangle_{k} / \sum_{k} \sigma_{k}^{4 \pi}$, where $k$ runs over all the $n_{b}$ bins corresponding to $x_{\ell}$.

The $\cos \phi_{h}$ moments from hydrogen and deuterium data are shown in figure 2 as projections versus the relevant kinematic variables for both hadron charges. Both hydrogen and deuterium data show similar behavior: the $\left\langle\cos \phi_{h}\right\rangle$ moments are found to be sizable and negative for positive hadrons. The signal increases with $P_{h \perp}$ and with the hadron energy fraction $z$, except in the very high $z$ range, where the partonic interpretation of the cross section is no longer valid ${ }^{2}$. The signal for the negative hadrons is significantly lower, but the dependence versus $\mathrm{z}$ and $P_{h \perp}$ exhibits similar features.

Figure 3 shows the $\cos 2 \phi_{h}$ moments that are found to be slightly negative for positive hadrons, and slightly positive for negative hadrons. Different results for positive and negative hadrons are not unexpected because both experimental evidence and theoretical models predict opposite Boer-Mulders contributions for differently charged hadrons.

\section{REFERENCES}

1. A. Bacchetta et al., JHEP 0702,093 (2007).

2. R. N. Cahn, Phys. Lett. B 78, 269 (1978), R. N. Cahn, Phys. Rev. D 40, 3107 (1989).

3. D. Boer and P. J. Mulders, Phys. Rev. D 57, 5780 (1998).

4. K. Ackerstaff et al. (HERMES collaboration), Nucl. Instr. Meth. A 417, 230 (1998).

5. F. Giordano, Proceedings of Transversity 2008 workshop, May 28-31, 2008, Ferrara, Italy, to be published by World scientific.

6. G. Cowan, Statistical Data Analysis, Clarendon Press, Oxford, 1998.

2 The highest $z$-bin is plotted for completeness but it is not used for projecting moments onto the single variables. 